\title{
Study on Six Degree-of-Freedom Electromagnetic Tracking System
}

\author{
YIN Qin ${ }^{1, a}$, WANG Dong ${ }^{1,2, b}$, SHEN Xinmin ${ }^{1, c}$, YANG Chengsong ${ }^{1, d}$ \\ ${ }^{1}$ College of Field Engineering, Army Engineering University, Nanjing, 210014, China \\ ${ }^{2}$ Second Institute of Engineering Research and Design, Southern Theater Command, Kunming, \\ 650222, China. \\ adfyq1982@21cn.com, bdyhkxydfbb@163.com, cshenxmifilgdx2014@163.com, \\ dycsdongshang@163.com
}

\begin{abstract}
Keywords: Electromagnetic Tracking; Anisotropic Parameters; Eigenvector; Tracking Algorithm. Abstract: In order to solve the problem of precision degrading caused by the different gains of three axes in six degree of freedom electromagnetic tracking system, the model of anisotropic parameters was introduced, based on magnetic dipole model. An improved arithmetic was proposed to calculate the location parameters, which was a combination of the maximal ponderance of unit pointing vector tracking arithmetic and the eigenvector arithmetic. This arithmetic solved the problem of magnifying calculation error in some angle area. The orientation transformation matrix can be calculated by received matrix, which avoids the error accumulation. These methods simplified the complexity and promoted the system stability. An new method to get the anisotropic parameters was estabished by three knowd points. The numeriacl simualtions and experiment results prove the model is valid and can improve the precision of the system.
\end{abstract}

\section{Introduction}

Six degree-of-freedom electromagnetic tracking system is a new kind of location and orientation tracking system, which can track six parameters of a target. This system has been wildly applied in airborne fire control system ${ }^{[1]}$, precise medical instruments ${ }^{[2]}$, virtual reality systems ${ }^{[3]}$, simulation training ${ }^{[4-5]}$, and location detecting ${ }^{[6]}$. Electromagnetic tracking system has the advantage over the other space locating methods, since it has no damage, no interference with the line of sight, and it could operate conventionally. There are two types of excitation sources emitting the magnetic field. One is alternating current (AC) field and the other is pulsed direct current (DC) field. By now, AC field has a more widespread application using the magnetic core coils as the sensors. This system is usually composed of three parts: a transmitting coils which generates a magnetic field, a receiving sensor which detects the field and a process. The transmitting and receiving sensor are both 3 magnetic core coils oriented orthogonal to each other.

The traditional tracking models are all based on the assumption that the parameters of three axes are approximately the same value in three directions of the coils. However, the parameters, including the area and turn number of the coils, the amplitude and frequency of the exciting current, and the system gain etc., are different in three direction, which affect the accuracy of the system seriously. In order to solve the problem of precision degrading caused by the different gains of three axes, the model of anisotropic parameters is introduced. The anisotropic parameters could be obtained by the coil numbers, coil area, current magnitude and frequency, and circuit gain. Electromagnetic tracking model with anisotropic parameters is proposed based on magnetic dipole antennas near filed model. An improved arithmetic is proposed to calculate the location parameters, which is a combination of the maximal ponderance of unit pointing vector tracking arithmetic and the eigenvector arithmetic, and solves the problem of magnifying calculation error in some angle area. The method to get the anisotropic parameters is also proposed base on the receiving matrix of three known location. At last, the numerical simulations and experiment results prove the accuracy of proposed model and algorithm. 


\section{Tracking Model}

The principle of the system is as follows ${ }^{[7]}$ : the antenna used is a three-axis loop antenna. The geometric relationship is shown in Fig.1. The receiving antenna's location is specified by $R$ (the distance), $\alpha$ (the azimuth angle), $\beta$ (the elevation angle), and its orientation is specified by $\psi$ (the yaw angle), $\theta$ (the pitch angle) and $\Phi$ (the roll angle). When the system works, all transmitting coils are excited by signals of the same frequency and phase. The excitation pattern is composed of three sequential excitation states. The receiving pattern is composed of three simultaneous states. So during one period of excitation, the receiving antenna receives nine signals which form the receiving matrix.

The anisotropic parameters are decided by the system hardware, including these influences such as the coil numbers, coil area, current magnitude and frequency, and circuit gain. We defined these parameters as follows:

$$
\begin{gathered}
C_{i}=\frac{\mu N_{i} I_{i} S_{i}}{4 \pi} \quad(i=x, y, z) \\
K_{j}=\omega n_{j} g_{j} S_{j} \quad(j=x, y, z)
\end{gathered}
$$

Where:

$\mu$ : magnetic permittivity

$N_{i} 、 n_{i}$ : the turn number of transmitting and receiving coils in $i$ axis;

$S_{i} 、 s_{i}$ : the area of transmitting and receiving coils in $i$ axis;

$I_{i} 、 \omega$ : the amplitude and frequency of exciting current in $i$ axis;

$g_{j}:$ the system gain of $j$ axis 。

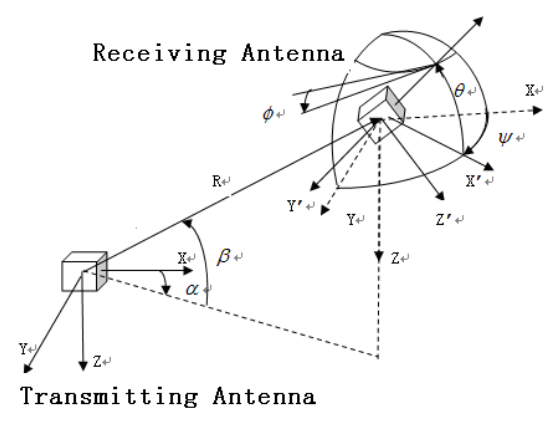

Fig.1 Location and Orientation Coordinates

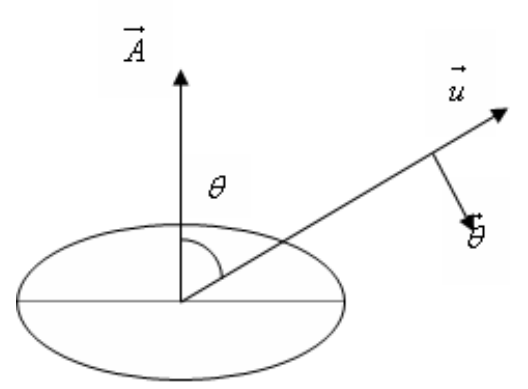

Fig.2 Sketch of Magnetic Dipole Model

According to the magnetic dipole model, the magnetic field of the transmitting coils can be expressed as ${ }^{[7,8]}$ :

$$
\stackrel{\text { ur }}{B_{i}}=\frac{\mu N_{i} I_{i} S_{i}}{4 \pi}\left[\frac{2 \cos \theta}{R^{3}} u_{i}+\frac{\sin \theta}{R^{3}} \boldsymbol{\bigotimes}_{i}\right]
$$

Exposing the component of $\$$ in $\$ A^{A}$ direction, we can get:

$$
\stackrel{\text { ur }}{B}_{i}=\frac{\mu N_{i} I_{i} S_{i}}{4 \pi}\left[-\frac{1}{R^{3}}\left(I_{3 \times 3}-3 u u\right) \mathbf{r}_{i}\right]
$$

So, the amplitude of the magnetic field near the receiving antenna can be expressed as:

$$
B_{i}=-\frac{\mu N_{i} I_{i} S_{i}}{4 \pi R^{3}} P M
$$

Where, $P$ is the orientation transformation matrix, $M$ is the location transformation matrix.

According to the Faraday's magnetic-electric inductive law, the amplitude of output voltage signal of the receiving coils in $j$ axis which is excited by transmitting coils in $i$ axis can be written:

$$
\begin{aligned}
S_{i j} & =-\frac{\mu N_{i} I_{i} S_{i}}{4 \pi R^{3}} P M \cdot \omega g_{j} n_{j} s_{j} \\
& =-C \cdot \frac{P M}{R^{3}} \cdot K \quad(i, j=x, y, z)
\end{aligned}
$$


Where: $S 、 P 、 M$ is $3 \times 3$ matrix.

$$
\begin{aligned}
& \$=\left[\begin{array}{l}
u_{1} \\
u_{2} \\
u_{3}
\end{array}\right]=\left[\begin{array}{c}
\sin \beta \cos \alpha \\
\sin \beta \sin \alpha \\
\cos \beta
\end{array}\right] \\
& C=\left[\begin{array}{ccc}
\frac{\mu N_{x} I_{x} S_{x}}{4 \pi} & 0 & 0 \\
0 & \frac{\mu N_{y} I_{y} S_{y}}{4 \pi} & 0 \\
0 & 0 & \frac{\mu N_{z} I_{z} S_{z}}{4 \pi}
\end{array}\right] \\
& \mathrm{M}=\left[\begin{array}{ccc}
1-3 \sin ^{2} \beta \cos ^{2} \alpha & -3 \sin ^{2} \beta \cos \alpha \sin \alpha & -3 \sin \beta \cos \alpha \cos \beta \\
-3 \sin ^{2} \beta \cos \alpha \sin \alpha & 1-3 \sin ^{2} \beta \sin ^{2} \alpha & -3 \sin \beta \sin \alpha \cos \beta \\
-3 \sin \beta \cos \alpha \cos \beta & -3 \sin \beta \sin \alpha \cos \beta & 1-3 \cos ^{2} \beta
\end{array}\right] \\
& K=\left[\begin{array}{ccc}
\omega g_{x} n_{x} s_{x} & 0 & 0 \\
0 & \omega g_{y} n_{y} s_{y} & 0 \\
0 & 0 & \omega g_{z} n_{z} s_{z}
\end{array}\right] \\
& P=\left[\begin{array}{ccc}
\cos \psi \cos \theta & \sin \psi \cos \theta & -\sin \theta \\
\cos \psi \sin \theta \sin \phi-\sin \psi \cos \phi & \sin \psi \sin \theta \sin \phi+\cos \psi \cos \phi & \sin \phi \cos \theta \\
\cos \psi \sin \theta \cos \phi+\sin \psi \sin \phi & \sin \psi \sin \theta \cos \phi-\cos \psi \sin \phi & \cos \theta \cos \phi
\end{array}\right]
\end{aligned}
$$

\section{Algorithm of Location Parameters}

In order to solve the problem of magnifying calculation error in some angle area, the maximal ponderance of unit pointing vector tracking arithmetic is proposed combinating with teh eigenvector arithmetic.

From the equations (6), we can get:

$$
\left(C^{-1} \cdot S_{i j} \cdot K^{-1}\right)^{\mathrm{T}}\left(C^{-1} \cdot S_{i j} \cdot K^{-1}\right)=\left(\frac{P M}{R^{3}}\right)^{\mathrm{T}}\left(\frac{P M}{R^{3}}\right)
$$

Since: $P^{T}=P^{-1}, M^{T}=M$, then equation (7) can be written as:

$$
\begin{aligned}
& \left(C^{-l} \cdot S_{i j} \cdot K^{-I}\right)^{\mathrm{T}}\left(C^{-l} \cdot S_{i j} \cdot K^{-I}\right)=\frac{M^{2}}{R^{6}} \\
& =\frac{1}{R^{6}}\left[\begin{array}{ccc}
1+3 \sin ^{2} \beta \cos ^{2} \alpha & 3 \sin ^{2} \beta \cos \alpha \sin \alpha & 3 \sin \beta \cos \alpha \cos \beta \\
3 \sin \beta \cos \alpha \sin \alpha & 1+3 \sin ^{2} \beta \sin 2 & 3 \\
3 \sin \beta \cos \beta & 3 \sin \beta \sin \alpha \cos \beta & 1+3 \cos ^{2} \beta
\end{array}\right]
\end{aligned}
$$

Set: $Y_{i j}=\left(C^{-1} \cdot S_{i j} \cdot K^{-1}\right)^{\mathrm{T}}\left(C^{-1} \cdot S_{i j} \cdot K^{-1}\right)$, so $w=Y_{11}+Y_{22}+Y_{33}=\frac{6}{R^{6}}$, then the distance can be calculated:

$$
R=\sqrt[6]{\frac{6}{w}}
$$

From equation (8), we can get:

$$
\frac{4}{R^{6}} \$=Y \$
$$

Set: $\quad \lambda=\frac{4}{R^{6}}$, we can get: $\quad\left(Y_{i j}-\lambda \bar{I}_{3 \times 3}\right) \$=0$

obviously, the unit pointing vector $\$$ is the eigenvector of matrix $\mathrm{Y}$ about $\lambda$, so:

$$
\lambda=\frac{4}{R^{6}}=\frac{2}{3} w=\frac{2}{3}\left(Y_{11}+Y_{22}+Y_{33}\right)
$$

According to the characteristics of eigenvector, we can get:

$$
\begin{aligned}
& u_{1}=l_{p 1} h \\
& u_{2}=l_{p 2} h \\
& u_{3}=l_{p 3} h
\end{aligned}
$$

Where: ${ }^{l}{ }$ is the algebraic complement of the matrix $Y_{i j}-\lambda I_{3 \times 3}$,

set: $\quad h= \pm \max \left(\frac{1}{\sqrt{l_{p 1}^{2}+l_{p 2}^{2}+l_{p 3}^{2}}}\right) \quad p=1,2,3$ vector tracking arithmetic ${ }^{[9]}$, we can get:

, according to the maximal ponderance of unit pointing

$$
\begin{aligned}
& \beta=\cos ^{-1}\left(u_{3}\right) \\
& \alpha=\cos ^{-1}\left[\frac{u_{1}}{\sin (\beta)}\right]
\end{aligned}
$$




\section{Algorithm of Orientation Parameters}

Since: $M^{-1}=\frac{3 I_{3 \times 3}-M^{2}}{2}$, from the equation (8), we can get:

$$
M^{2}=\frac{6}{w} Y_{i j}
$$

So:

$$
M^{-1}=\frac{\left[3 I_{3 \times 3}-\frac{6}{w} Y_{i j}\right]}{2}
$$

Then, the orientation transformation matrix $\mathrm{P}$ can be calculated:

$$
P=-\sqrt{\frac{6}{w}} \cdot\left[C^{-1} \cdot S_{i j} \cdot K^{-1}\right] \cdot \frac{\left[3 I_{3 \times 3}-\frac{6}{w} Y_{i j}\right]}{2}
$$

Therefore, we can calculate the orientation parameters based on tracking arithmetic ${ }^{[9]}$ :

$$
\begin{aligned}
& \psi=\tan ^{-1}\left(\frac{P_{12}}{P_{11}}\right) \\
& \theta=\sin ^{-1}\left(-P_{13}\right) \\
& \phi=\tan ^{-1}\left(\frac{P_{23}}{P_{33}}\right)
\end{aligned}
$$

\section{Algorithm of anisotropic parameters}

A simple method to get the anisotropic parameters of system was proposed ${ }^{[10]}$. This method obtained the parameters by measuring the magnetic field on three different points. The plane of the points was perpendicular to the axis of the transmitting coil. The transmitting and receiving system parameters were calculated individually, and a magnetic sensor was need additionally. We propose an improved method to calculate the anisotropic parameters of system simultaneously, and need no additional magnetic sensor.

The coordinate system $X Y Z$ can be established as Fig.3, the core of transmitting coils coordinates $\operatorname{are}^{(\Delta x, \Delta y, \Delta z)}$, set $P_{0} 、 P_{1} 、 P_{2}$ as $(0, b, 0),(a, b, 0),(-a, b, 0)$, where: $a>\Delta x>0 、 b>\Delta y>0$, so the coordinates of $P_{0} 、 P_{1} 、 P_{2}$ can be written as : $(-\Delta x, b-\Delta y,-\Delta z),(a-\Delta x, b-\Delta y,-\Delta z)$, $(-a-\Delta x, b-\Delta y,-\Delta z)$ 。

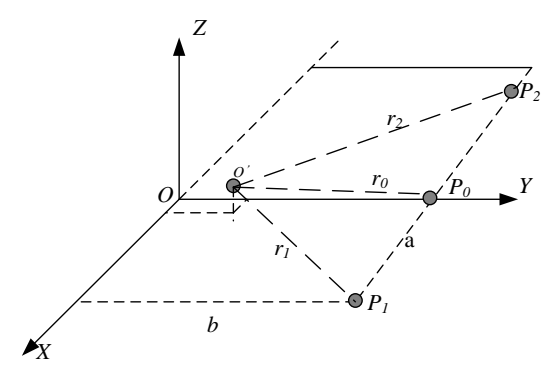

Fig.3 diagrammatic sketch of measurement points

According to the coordinates systems, we can get:

$$
\begin{aligned}
& r_{0}=\left|P_{0} O^{\prime}\right|=\sqrt{\Delta x^{2}+(b-\Delta y)^{2}+\Delta z^{2}} \\
& r_{1}=\left|P_{1} O^{\prime}\right|=\sqrt{(a-\Delta x)^{2}+(b-\Delta y)^{2}+\Delta z^{2}}=\sqrt{a^{2}-2 a \Delta x+r_{0}^{2}} \\
& r_{2}=\left|P_{2} O^{\prime}\right|=\sqrt{(a+\Delta x)^{2}+(b-\Delta y)^{2}+\Delta z^{2}}=\sqrt{a^{2}+2 a \Delta x+r_{0}^{2}}
\end{aligned}
$$

Based on magnetic dipole model and Faraday's magnetic-electric inductive law, the receiving matrix at point $P_{0}$ are as follows: 


$$
\left[\begin{array}{ccc}
u_{0 x x} & u_{0 y x} & u_{0 z x} \\
u_{0 x y} & u_{0 y y} & u_{0 z y} \\
u_{0 x z} & u_{0 y z} & u_{0 z z}
\end{array}\right]=\left[\begin{array}{ccc}
C_{x} K_{x}\left(\frac{3 \Delta x^{2}}{r_{0}^{5}}-\frac{1}{r_{0}^{3}}\right) & -C_{y} K_{x} \frac{3 \Delta x(b-\Delta y)}{r_{0}^{5}} & C_{z} K_{x} \frac{3 \Delta x \Delta z}{r_{0}^{5}} \\
-C_{x} K_{y} \frac{3(b-\Delta y) \Delta x}{r_{0}^{5}} & C_{y} K_{y}\left(\frac{3(b-\Delta y)^{2}}{r_{0}^{5}}-\frac{1}{r_{0}^{3}}\right) & -C_{z} K_{y} \frac{3(b-\Delta y) \Delta z}{r_{0}^{5}} \\
C_{x} K_{z} \frac{3 \Delta x \Delta z}{r_{0}^{5}} & -C_{y} K_{z} \frac{3(b-\Delta y) \Delta z}{r_{0}^{5}} & C_{z} K_{z}\left(\frac{3 \Delta z^{2}}{r_{0}^{5}}-\frac{1}{r_{0}^{3}}\right)
\end{array}\right]
$$

We can also get another two matrix at points $P_{1}$ and $P_{2}$, from these matrixes, we can calculated the anisotropic parameters. They can be expressed as follows:

$$
\begin{array}{lll}
C_{x} K_{x}=\frac{\left|u_{0 x x}\right| r_{0}^{5}}{\left|3 \Delta x^{2}-r_{0}^{2}\right|} & C_{y} K_{x}=\frac{\left|u_{0 y x}\right| r_{0}^{5}}{3 \Delta x(b-\Delta y)} & C_{z} K_{x}=\frac{\left|u_{0 z x}\right| r_{0}^{5}}{3 \Delta x \Delta z} \\
C_{x} K_{y}=\frac{\left|u_{0 x y}\right| r_{0}^{5}}{3(b-\Delta y) \Delta x} & C_{y} K_{y}=\frac{\left|u_{0 y y}\right| r_{0}^{5}}{\left|3(b-\Delta y)^{2}-r_{0}^{2}\right|} & C_{z} K_{y}=\frac{\left|u_{0 z y}\right| r_{0}^{5}}{3(b-\Delta y) \Delta z} \\
C_{x} K_{z}=\frac{\left|u_{0 x z}\right| r_{0}^{5}}{3 \Delta z \Delta x} & C_{y} K_{z}=\frac{\left|u_{0 y z}\right| r_{0}^{5}}{3(b-\Delta y) \Delta z} & C_{z} K_{z}=\frac{\left|u_{0 z z}\right| r_{0}^{5}}{\left|3 \Delta z^{2}-r_{0}^{2}\right|}
\end{array}
$$

\begin{tabular}{|c|c|c|c|c|c|c|}
\hline \multicolumn{6}{|c|}{ anisotropic parameters } & isotropic parameters \\
\hline$C_{x} K_{x}$ & 299.76 & $C_{y} K_{x}$ & 356.78 & $C_{z} K_{x}$ & 400.54 & \multirow{3}{*}{362.36} \\
\hline$C_{x} K_{y}$ & 308.91 & $C_{y} K_{y}$ & 367.66 & $C_{z} K_{y}$ & 412.76 & \\
\hline$C_{x} K_{z}$ & 316.12 & $C_{y} K_{z}$ & 376.25 & $C_{z} K_{z}$ & 422.40 & \\
\hline
\end{tabular}

\section{Numerical Simulations and Experiments}

The magnetic field of the transmitting coils can be simulated by the parallel-FDTD. Anisotropic parameters can be determined by the turns and area of coils, exciting current and the system gains. Isotropic parameter is the average. Those parameters are in table 1. The numerical results are shown in table 2.

Table 1: parameters of numerical simulations

Table 2: numerical simulation results

\begin{tabular}{|l|l|l|l|l|l|l|}
\hline Station 1 & $R(\mathrm{~m})$ & $\alpha\left(^{\circ}\right)$ & $\beta\left(^{\circ}\right)$ & $\psi\left(^{\circ}\right)$ & $\theta\left(^{\circ}\right)$ & $\Phi\left(^{\circ}\right)$ \\
\hline Theoretical value & 0.30 & 30.00 & 45.00 & 10.00 & 30.00 & 45.00 \\
\hline Isotropic model & 0.29 & 37.61 & 38.39 & 13.42 & 10.86 & 49.79 \\
\hline Anisotropic model & 0.30 & 30.00 & 46.28 & 10.27 & 29.19 & 45.82 \\
\hline Station 2 & $R(\mathrm{~m})$ & $\alpha\left(^{\circ}\right)$ & $\beta\left(^{\circ}\right)$ & $\psi\left(^{\circ}\right)$ & $\theta\left(^{\circ}\right)$ & $\Phi\left(^{\circ}\right)$ \\
\hline Theoretical value & 0.40 & 60.00 & 130.00 & -10.00 & 120.00 & 60.00 \\
\hline Isotropic model & 0.39 & 65.95 & 134.06 & 2.01 & 119.60 & 89.57 \\
\hline Anisotropic model & 0.39 & 59.34 & 130.56 & -11.41 & 118.74 & 62.05 \\
\hline
\end{tabular}

In system experiments, the anisotropic parameters are calculated by the method above, as in Table

3. The results are shown in table4.

Table 3: parameters of experiments

\begin{tabular}{|l|c|l|l|l|l|l|}
\hline \multicolumn{2}{|c|}{ anisotropic parameters } & isotropic parameters \\
\hline$C_{x} K_{x}$ & 293 & $C_{y} K_{x}$ & 299 & $C_{z} K_{x}$ & 308 & \multirow{2}{*}{321} \\
\hline$C_{x} K_{y}$ & 313 & $C_{y} K_{y}$ & 319 & $C_{z} K_{y}$ & 329 & \\
\hline$C_{x} K_{z}$ & 333 & $C_{y} K_{z}$ & 340 & $C_{z} K_{z}$ & 351 & \\
\hline
\end{tabular}


Table 4: experiment results (azimuth angle $\alpha$ )

\begin{tabular}{|c|c|c|c|c|}
\hline station & Theoretical value & Isotropic model & Anisotropic model & Increased accuracy \\
\hline 1 & 10 & 6.75 & 9.1 & $23.5 \%$ \\
\hline 2 & 20 & 15.6 & 18 & $12 \%$ \\
\hline 3 & 30 & 24.8 & 30.1 & $17.67 \%$ \\
\hline 4 & 40 & 34.2 & 41 & $17 \%$ \\
\hline 5 & 50 & 54.1 & 50.3 & $7.6 \%$ \\
\hline 6 & 60 & 64.3 & 61 & $5.5 \%$ \\
\hline
\end{tabular}

\section{Conclusions}

An improved tracking model is proposed utilizing the anisotropic parameters, which is more accordant to the nature of electromagnetic tracking system. The location and orientation information of target can be calculated by the maximal ponderance of unit pointing vector and the tracking arithmetic. The anisotropic parameters of system can be deduced by a new method using three known points. The numerical simulations and experimental results prove the algorithm is valid and the accuracy of the system has been improved.

\section{Acknowledgements}

This work is financially supported by the National Natural Science Foundation of China(51505498、41401518) and Natural Science Foundation of Jiangsu Province (BK20150714)

\section{References}

[1] Sheng-Jen Hsieh, Clarence E. Rash, Thomas H, et al. Helmet-Mounted Display Image Quality Evaluation System [J]. IEEE Trans. on, Instrumentation and Measurement, 2003, 52(6): 1838-1845.

[2] Kevin Cleary, Hui Zhang, Neil Glossop, et al. Electromagnetic Tracking for Image-Guided Abdominal Procedures: Overall System and Technical Issues[C]// Proc. of the 2005 IEEE Engineering in Medicine and Biology 27th Annual Conference. Shanghai, IEEE press, 2005: 6748-6753.

[3] Xu X F. Dynamic Organization and Methodology for Agile Virtual Enterprises [J]. Journal of Computer Science and Technology, 2000, 15(4): 368-375.

[4] T. Hollerer, S. Feiner, T. Terauchi, et al. Exploring MARS: Developing Indoor and Outdoor User Interfaces to a Mobile Augmented Reality System [J]. Computers \& Graphics, 1999, 23(6): 779-785.

[5] R. Tenmoku, M. Kanbara, N. Yokoya. A Wearable Augmented Reality System Using Positioning Infrastructures and a Pedometer [C]//Proc. of 7th IEEE Symposium on Wearable Computers, New York, IEEE Compute Society, 2003:110-117.

[6] Anon. Measuring bending stress in buried metal gas pipes [J]. Journal of Failure Analysis and Prevention, 2004, 4 (4): 6-7.

[7] Chen Bin. Analysis and Research of the Electromagnetic Sensing System of Helmet-mounted Sighting Units[D]. The dissertation of Beijing University of Science and Technology,1988.3:10-18.

[8] Yin Qin, Chen Bin, Wang Ying, et.al. Six degree of freedom electromagnetic tracking system based on anisotropic parameters model[J]. Systems Engineering-Theory \& Practice, 2011, Vol.31,No.2:351-356.

[9] Yin Qin, Chen Bin, etal. Research on Tracking Arithmetic of Six-stance Electromagnetic Sensitivity Position System[C]. IEEE 2007 International Symposium on Microwave, Antenna, 
Propagation and EMC Technologies for Wireless Communications, Hangzhou : Institute of Electrical and Electronics Engineers, Inc.2007,2(2):1433-1436.

[10] Yan Zhi-gang, Yuan Kui. Simple Method to Get Parameters of Magnetic Tracking System[J]. Journal of System Simulation, 2007, Vol.19, No.1:81:85. 